\title{
Trends in Medicaid spending on inhalers in the United States, 2012-2018
}

\author{
Farideh Sistani, PharmD; Robert M Reed, MD; Chintal H Shah, MS; and Zafar Zafari, MSc, PhD
}

\section{What is already known about this subject}

- Respiratory diseases and expenditures for inhalers impose a significant economic burden on Medicaid.

- In 2010, Medicaid paid 25\% of medical costs attributed to chronic obstructive pulmonary disease (COPD), which endured the highest cost burden after Medicare (51\%).

\section{What this study adds}

- Because inhaler maintenance therapy is the mainstay treatment for alleviating symptoms and reducing rates of exacerbations in COPD and asthma patients, it is critical to understand the direct economic burden of inhalers on Medicaid.

- This study reports on Medicaid spending trends on inhalers from 2012 to 2018 in the United States.

\section{Author affiliations}

Farideh Sistani, PharmD; Chintal H Shah, MS; and Zafar Zafari, MSc, PhD, Pharmaceutical Health Services Research, University of Maryland School of Pharmacy, Baltimore. Robert M Reed, MD, Associate Professor of Medicine, University of Maryland School of Medicine, Baltimore.

\section{AUTHOR CORRESPONDENCE:}

Zafar Zafari, 410.706.5740;

zzafari@rx.umaryland.edu

J Manag Care Spec Pharm. 2021;27(12):1744-49

Copyright $\left(C_{2021}\right.$, Academy of Managed Care Pharmacy. All rights reserved.

the inhaler class of ICS/long-acting beta-2 adrenoceptor agonists contributed to the highest Medicaid spending (53\%), with a growth of $607 \%$ between 2012 and 2018 . Of the $\$ 26.2$ billion that Medicaid spent on inhalers, $\$ 35.5$ million (less than $0.01 \%$ ) was spent on 2 generic inhalers: fluticasone propionate with salmeterol and levalbuterol tartrate hydrofluoroalkane.

RESULTS: Medicaid spent $\$ 26.2$ billion on inhalers from 2012 to 2018. This spending increased by $\$ 2.5$ billion ( $120 \%$ ) over this time frame. During this specified period, the highest Medicaid spending was on the group of inhaled corticosteroid (ICS)-containing inhalers (\$14.9 billion). Within this group,
CONCLUSIONS: Between 2012 and 2018, on average, $\$ 3.5$ billion per year was spent by Medicaid on inhalers. Decreasing the price of inhalers by introducing more generic inhalers in the market can potentially reduce the cost burden on Medicaid. 
Chronic obstructive pulmonary disease (COPD) and asthma are complex respiratory diseases that are associated with chronic inflammation of the respiratory airways. ${ }^{1}$ In the United States, more than 16 million people have been diagnosed with COPD. ${ }^{2}$ In 2019, 8\% of Americans aged 18 years and over, and $7 \%$ of children younger than 18 years were diagnosed with asthma. ${ }^{3}$

Despite the significant health and economic toll that COPD and asthma take on society, ${ }^{4,5}$ to date, there is no definite cure for these conditions. Nevertheless, there are effective inhalers to subdue symptoms of these respiratory diseases if patients stay adherent to them. ${ }^{6}$

Medicaid is a joint state and federal program that provides health coverage for low-income adults and individuals with disabilities. ${ }^{7}$ In 2010, Medicaid paid 25\% of medical costs attributed to COPD and endured the highest cost burden after Medicare (51\%). ${ }^{8}$ Likewise, Medicaid is a major source of insurance coverage for asthma patients. Adults aged 18-64 years under Medicaid have asthma at a prevalence of $13.1 \%{ }^{9}$ Since inhaler maintenance therapy is the mainstay treatment for alleviating symptoms and reducing rates of exacerbations in COPD and asthma patients,,$^{10}$ it is critical to understand Medicaid's spending on inhalers.

The purpose of this study was to describe the total spending on inhalers and trends in Medicaid expenditures for different classes of inhalers from 2012 to 2018. We also compared the spending on available generic inhalers with their brand medications. Furthermore, the inhaler market share is described for the top 4 manufacturers in terms of Medicaid spending.

\section{Methods}

\section{DATA}

We queried the publicly available Medicaid Drug Spending Dashboard from 2012 to 2018 to identify inhalers. The webbased tool includes datasets for medications paid through the Medicaid program. The deidentified datasets represent drug use and spending data at the national level for outpatient drugs that are paid through state Medicaid agencies. These datasets contain more than 9,000 different medications and medical devices indicated to treat various health conditions and provide information on aggregate Medicaid spending, drug types, and manufacturers. Medicaid drug spending metrics are composed of federal and state reimbursement, including any applicable dispensing fees. ${ }^{11}$

\section{ANALYSIS}

We identified 9 classes of inhalers according to their active ingredients based on the Global Initiative for Chronic
Obstructive Lung Disease (GOLD) and the Global Initiative for Asthma (GINA) guidelines. ${ }^{12,13}$ We categorized these inhalers into 3 main groups: (1) short-acting inhalers, including short-acting beta-2 adrenoreceptor agonists (SABA), shortacting muscarinic agents (SAMA), and the combination of SAMA/SABA; (2) long-acting inhalers, including long-acting beta-2 adrenoceptor agonists (LABA), long-acting muscarinic agents (LAMA), and the combination of LABA/LAMA; and (3) inhaled corticosteroids (ICS)-containing inhalers, including ICS, ICS/LABA, and ICS/LAMA/LABA.

We pooled estimates from Medicaid Drug Spending Dashboard datasets to calculate the overall spending on inhalers. We calculated the total and trends of spending by each group (ie, ICS-containing, short-acting, and longacting groups of inhalers) and within each group by each class of inhalers. We also pooled data on the manufacturers and quantified the Medicaid spending by different manufacturers over the 7-year study period.

Calculation of spending was adjusted for inflation to 2018 US dollars using a 3\% annual discount rate. ${ }^{14}$ All analyses were performed in the statistical software Python. ${ }^{15}$ No institutional review board approval was needed, since only publicly sourced expenditure data were used for our analyses. Also, patient-level data were not accessed in this study.

\section{Results}

We identified and analyzed 44 (42 brand and 2 generic) inhaler medications covered by Medicaid. For the group of ICS-containing inhalers, we analyzed 7 ICS (ciclesonide, fluticasone propionate, fluticasone furoate, mometasone furoate, budesonide, flunisolide, and beclomethasone dipropionate); 4 ICS/LABA (mometasone with formoterol, fluticasone propionate with salmeterol, fluticasone with vilanterol, and budesonide with formoterol fumarate); and 1 ICS/LAMA/LABA (fluticasone with umeclidinium and vilanterol). For the group of long-acting inhalers, we analyzed 4 LABA (salmeterol xinafoate, indacaterol maleate, olodaterol HCL, and formoterol fumarate); 3 LAMA (tiotropium bromide, umeclidinium bromide, and aclidinium bromide); and 4 LABA/LAMA (indacaterol with glycopyrrolate, umeclidinium bromide with vilanterol trifentate, glycopyrrolate with formoterol fumarate, and tiotropium bormide with olodaterol hydrochloride). For the group of short-acting inhalers, we analyzed 2 SABA (albuterol sulfate and levalbuterol tartrate); 1 SAMA (ipratropium bromide); and 1 SAMA/SABA (ipratropium with albuterol sulfate).

\section{TRENDS OF OVERALL MEDCAID SPENDING}

Over the 7-year study period (2012-2018), the total Medicaid spending on inhalers was $\$ 26.2$ billion (6\% of Medicaid 


\section{FIGURE 1 Trend of Overall Medicaid Spending on Inhalers From 2012 to 2018}

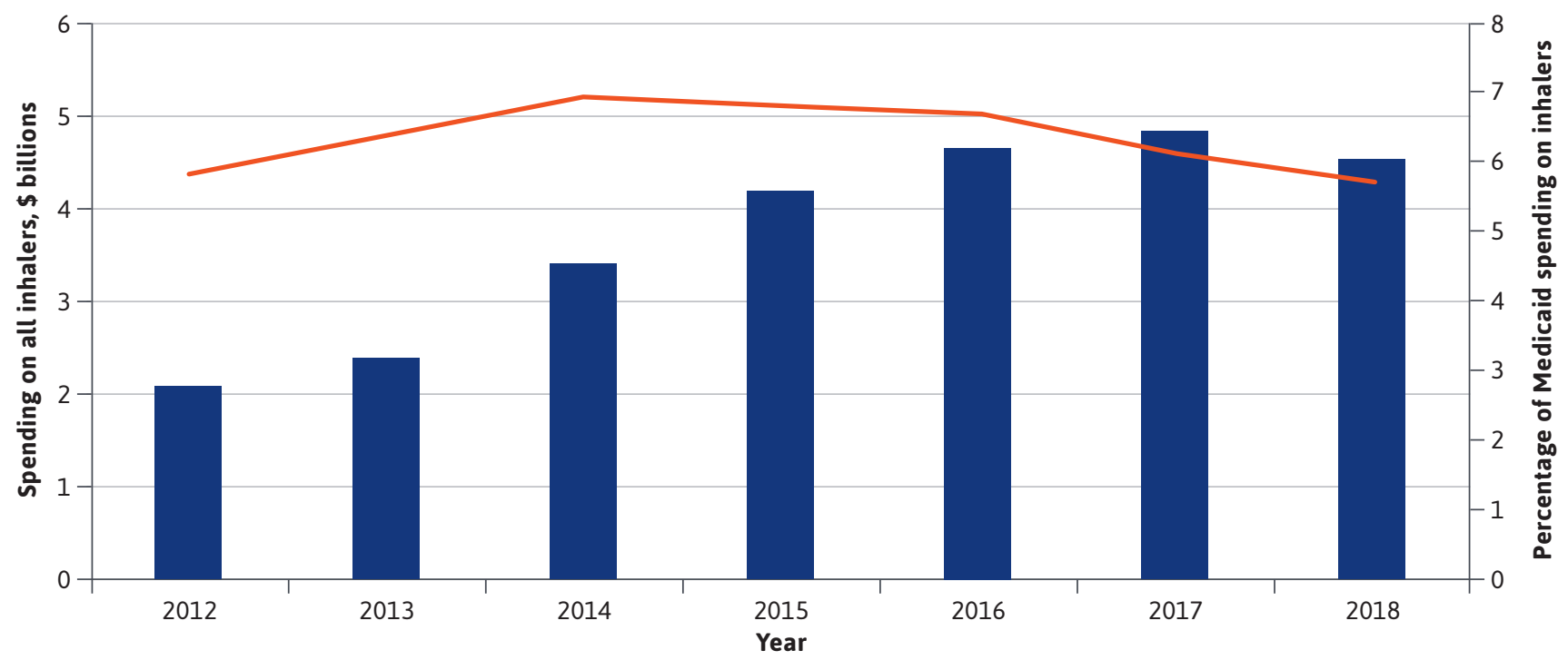

- Spending on inhalers

- Spending on inhalers as percentage of spending on all drugs

spending on all drugs). There was an increasing trend of spending on inhalers by Medicaid during the specified period. The total Medicaid spending on inhalers was \$2.1 billion in 2012, which increased to $\$ 4.6$ billion in 2018 (Figure 1). For context, Medicaid spending on all drugs increased from \$35.3 billion in 2012 to $\$ 79.3$ billion in 2018 (Supplementary Figure 1, available in online article).

During the study period, the highest Medicaid spending on inhalers was $\$ 14.9$ billion (57\%) on ICS-containing inhalers, followed by $\$ 7.8$ billion (29.8\%) on short-acting inhalers and $\$ 3.5$ billion (13.2\%) on long-acting inhalers.

From 2012 to 2018, the highest growth of Medicaid spending was on ICS-containing inhalers (168\%), followed by long-acting inhalers (98\%) and short-acting inhalers (66\%; Figure 2).

Among ICS-containing inhalers, the highest spending was on the ICS/LABA class of inhalers (53\%), followed by the ICS class of inhalers (47\%). During the study period, Trelegy Ellipta (fluticasone with umeclidin and vilanterol) was the only ICS/LAMA/LABA inhaler covered by Medicaid. This inhaler, which was first marketed in 2017, comprised less than $0.04 \%$ of Medicaid spending on ICS-containing inhalers (Figure 2).

Among short-acting inhalers, $83 \%$ of the spending was on SABA inhalers. The classes of SAMA/SABA and SAMA inhalers contributed to $11 \%$ and $6 \%$ of the spending, respectively.

Among long-acting inhalers, the highest spending was on the class of LAMA inhalers (83\%), followed by the class of LABA inhalers (13\%) and the class of LABA/LAMA inhalers (4\%). The class of LABA/LAMA inhalers entered the market in 2014 (Figure 2).

\section{PRESCRIPTIONS FILLED OVER TIME}

From 2012 to 2018, the number of prescriptions filled for ICS-containing inhalers increased by $77 \%$, from 5.5 million prescriptions in 2012 to 9.8 million in 2018. The number of prescriptions filled for long-acting inhalers was 1.3 million in 2012 , which increased by $39 \%$ to 1.8 million in 2018 . For short-acting inhalers, the number of prescriptions filled in 2012 was 15 million, which increased by $18 \%$ to 17.7 million in 2018 (Supplementary Figure 2, available in online article).

\section{AVERAGE SPENDING PER SINGLE INHALER OVER TIME}

Over the study period, the average spending per single inhaler increased by $51 \%$ for ICS-containing inhalers, increased by $42 \%$ for long-acting inhalers, and increased by $40 \%$ for short-acting inhalers (Supplementary Figure 2). 


\section{FIGURE 2 Total Medicaid Spending per Inhaler Group, 2012-2018}
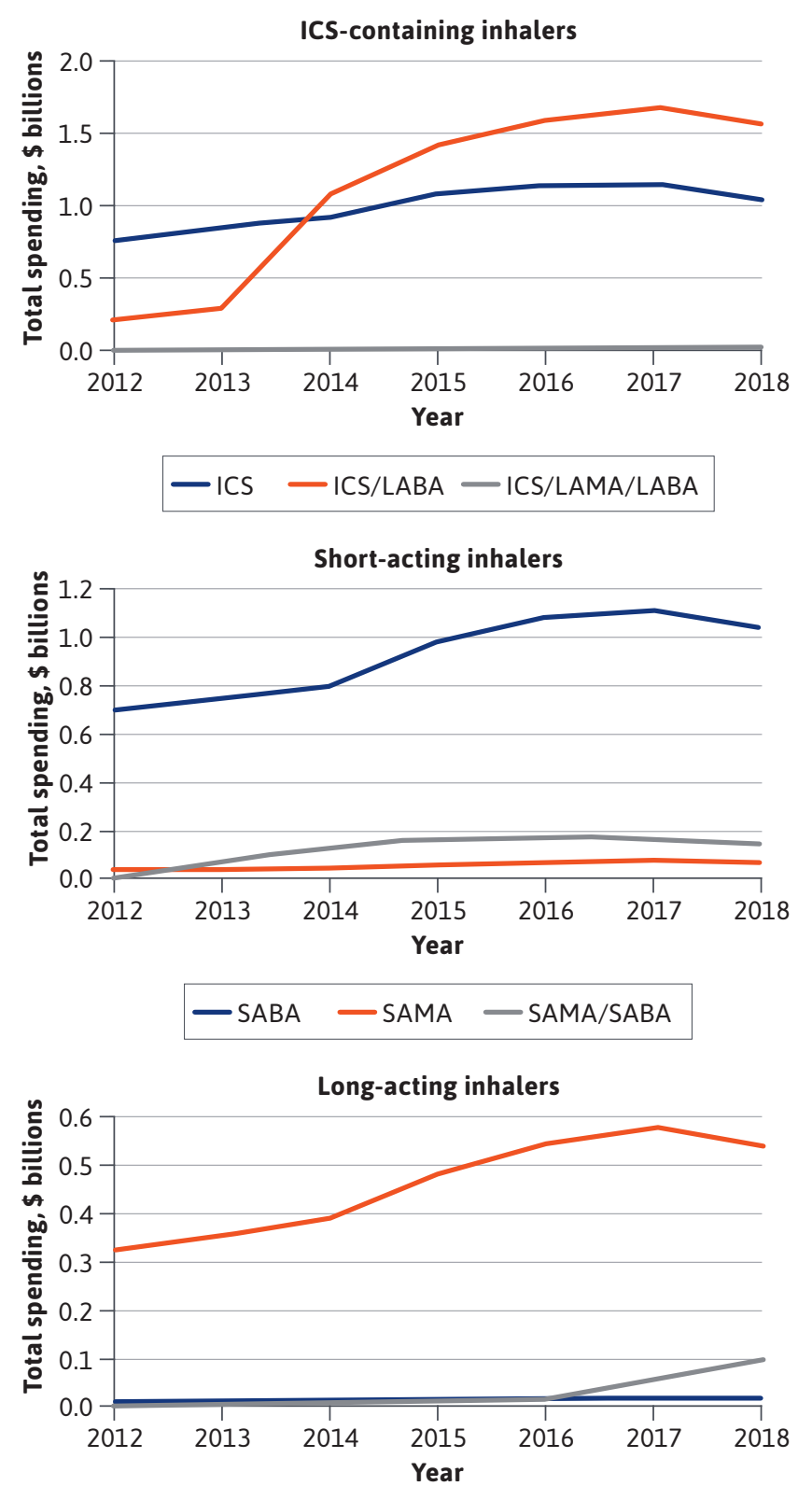

LABA - LAMA - LABA/LAMA

footer text

Vol. 27, No. 12 | December 2021 | JMCP.org

\section{MEDICAID SPENDING ON GENERIC VS BRAND INHALERS}

Medicaid spending between the years 2012 and 2015 was only on brand-name inhalers. Given that there are only a few generic inhalers available in the market, lower Medicaid spending on these inhalers was expected. The 2 generic inhalers in our study were first marketed in 2016, for generic SABA (levalbuterol tartrate hydrofluoroalkane [HFA]), and in 2017, for generic ICS/LABA (fluticasone propionate with salmeterol). From 2016 to 2018, Medicaid's spending on these generic inhalers was \$35.5 million (\$26.2 million on ICS/ LABA and $\$ 9.3$ million on SABA) compared with $\$ 14.1$ billion on brand inhalers. Overall, these generic inhalers contributed to $0.3 \%$ of the total Medicaid spending on inhalers between 2016 and 2018 .

\section{MEDICAID SPENDING BY MANUFACTURERS}

Between 2012 and 2018, the top 4 manufacturers for Medicaid-covered inhalers were GlaxoSmithKline, Teva Specialty, AstraZeneca, and Boehringer Ingelheim. The highest Medicaid spending (40\%) was on inhalers manufactured by GlaxoSmithKline, which produces the most classes of inhalers, including ICS-containing inhalers (ICS, ICS/LABA, ICS/LAMA/LABA), short-acting inhalers (SABA), and long-acting inhalers (LABA, LAMA/LABA, and LAMA). In contrast, Teva Specialty, AstraZeneca, and Boehringer Ingelheim contributed to $18 \%, 17 \%$, and $16 \%$ of Medicaid spending on inhalers, respectively. These manufacturers produce fewer classes of inhalers compared with GlaxoSmithKline. Teva Specialty produces mainly ICScontaining inhalers (ICS and ICS/LABA) and SABA inhalers. AstraZeneca produces ICS-containing inhalers (ICS and ICS/LABA) and long-acting inhalers (LAMA and LABA/ LAMA), and Boehringer Ingelheim mainly produces shortacting (SAMA and SAMA/SABA) and long-acting inhalers (LABA, LAMA, and LAMA/LABA; Supplementary Figure 3, available in online article).

\section{Discussion}

Our analyses demonstrated that the overall cost burden on Medicaid for inhalers increased by $120 \%$ from 2012 to 2018. This increase was slightly lower than the increase in Medicaid spending on all covered medications (125\%). The spending growth on inhalers was mostly attributable to ICS-containing inhalers (66\%) and less to short-acting inhalers (20\%) and long-acting inhalers (13\%).

Among ICS-containing inhalers, the highest growth of Medicaid spending belonged to ICS/LABA inhalers, which demonstrated an increase of 607\% between 2012 and 2018 . 
The increasing trend in the use of inhalers in this class has several potential explanations. One possibility is the increase in the number of filled prescriptions, which can be reflected by a rise in the number of beneficiaries with respiratory diseases, many of whom end up receiving this combination therapy to reduce risks of exacerbations. In a stepwise approach to asthma treatment, ICS/LABA inhalers are recommended for patients whose symptoms are not controlled with a low to moderate dose of mono ICS therapy. ${ }^{16}$ According to GOLD guidelines, ICS inhalers are reserved for COPD patients with frequent exacerbations, despite using combination therapy with LABA/LAMA. ${ }^{12}$

It is not always easy to differentiate between asthma and COPD. Physicians have difficulties in classifying up to $19.8 \%$ of patients with chronic respiratory symptoms into asthma or COPD groups. This uncertain diagnosis is, in part, responsible for the overuse of ICS inhalers in COPD patients. ${ }^{17}$ Thus, another possible explanation for the increased use of ICS/LABA inhalers could be a concern for asthma overlap in COPD patients among some physicians, which could contribute to a prescribing preference by them. LABA is considered as first-line therapy in the stepwise approach to COPD treatment ${ }^{10}$; in contrast, ICS is the treatment of choice indicated by the US Food and Drug Administration for the prevention of exacerbations in patients with persistent asthma. ${ }^{18}$ Another possibility that might explain the increase in Medicaid spending on ICS/LABA inhalers is the growth in spending per single inhaler over the study period. On average, the spending per single ICS/LABA inhaler increased from \$222 in 2012 to $\$ 317$ in 2018.

To calculate the proportion of growth in Medicaid spending on the ICS/LABA inhalers from 2012 to 2018 that is attributable to increases in spending per single inhaler over this time vs the attributable proportion to increases in the number of prescriptions filled over time, we calculated the projected growth when (1) the spending per single inhaler is held constant and (2) the number of prescriptions filled for this class is held constant. Our calculations showed that increased spending per single inhaler accounted for 35\% of the growth between 2012 and 2018, while the increased number of prescriptions accounted for $65 \%$ of the growth.

Despite such growth in Medicaid spending on ICS/ LABA inhalers over the time frame, the spending over the last 2 years (2017-2018) decreased by 6.3\%, potentially because of the arrival of the ICS/LABA generic inhaler into the market. Nevertheless, even with the availability of the fluticasone with salmeterol (ICS/LABA) generic inhaler on the market, brand-name inhalers still accounted for $97 \%$ of Medicaid's spending on this medication during 2017-2018. Similarly, the levalbuterol tartrate HFA (SABA) generic inhaler only accounted for $39 \%$ of Medicaid's spending on this medication during 2016-2018.

To the best of our knowledge, trends in Medicaid spending on inhalers have not yet been assessed in the United States. A recent similar study by Feldman et al analyzed trends of Medicare Part D inhaler spending..$^{19}$ According to that study, Medicare Part D spent $\$ 39.7$ billion on inhalers between 2012 and 2018. ${ }^{19}$ In comparison with our analysis, which showed a $\$ 2.5$ billion increase in Medicaid spending on inhalers from 2012 to 2018, such spending on inhalers by Medicare Part D only increased by $\$ 2$ billion over the same time period. ${ }^{19}$ In addition, according to Feldman et al, ICS/LABA inhalers had the highest contribution to the growth in Medicare Part D spending between 2012 and 2018. ${ }^{19}$ This finding is in line with our study, which showed that ICS/LABA inhalers accounted for $55 \%$ of the growth of Medicaid's spending on inhalers from 2012 to 2018.

\section{LIMITATIONS}

Our study faced some important limitations. The datasets that we used for our analyses from the Medicaid Drug Spending Dashboard lacked data on sociodemographic characteristics (eg, age, sex, and race); number of beneficiaries who received inhalers; and diagnostics codes, and we were not able to break down the inhalers' cost burden by these factors. In addition, the lack of individual-level data precluded us from analyzing expenditures by patientlevel characteristics. Nevertheless, our data sufficed for calculating aggregate estimates for overall and trends of Medicaid spending on inhalers. Finally, the costs reported did not account for rebates and discounts that might have been provided to states, and this should be accounted for while interpreting our results.

\section{Conclusions}

Our study provides population-level estimates and quantifies trends in Medicaid expenditures on inhalers. According to our analysis, while the average Medicaid spending on inhalers was $\$ 3.5$ billion per year, the spending increased from $\$ 2.1$ billion in 2012 to $\$ 4.6$ billion in 2018. Such growth can potentially reflect an increase in total Medicaid beneficiaries (from 58.9 million in 2012 to 73.9 million in 2018). ${ }^{20}$

As generic competition remains limited and brandname drug prices have continued to increase beyond the general inflation, ${ }^{21}$ introduction of more generic inhalers in the market may lower the price of inhalers and reduce the cost burden on Medicaid. Clinicians also should work to ensure accurate diagnosis and appropriate guidelinerecommended treatment for all asthma and COPD patients. 


\section{DISCLOSURES}

This study was funded by the American Foundation for Pharmaceutical Education (AFPE). The funders had no role in study design, data collection, and analysis, decision to publish, or preparation of the manuscript. The authors declare no conflicts of interest.

\section{REFERENCES}

1. Barnes Peter J. Cellular and molecular mechanisms of asthma and COPD. Clin Sci (Lond). 2017;131(13):1541-58. doi: 10.1042/ CS20160487.

2. Centers for Disease Control and Prevention. Chronic obstructive pulmonary disease (COPD). 2020. Updated February 22, 2021. Accessed October 10, 2021. https://www.cdc.gov/copd/index. html

3. Centers for Disease Control and Prevention. FastStats. Asthma. 2020. Updated August 23, 2021. Accessed October 10, 2021. https://www.cdc.gov/ nchs/fastats/asthma.htm

4. Zafari Z, Li S, Eakin MN, Bellanger M, Reed RM. Projecting long-term health and economic burden of COPD in the United States. Chest. 2021;159(4):1400-10. doi: 10.1016/j.chest.2020.09.255.

5. Yaghoubi M, Adibi A, Safari A, FitzGerald JM, Sadatsafavi M. The projected economic and health burden of uncontrolled asthma in the United States. Am J Respir Crit Care Med. 2019;200(9):1102-12.

6. Zafari Z, Lynd LD, FitzGerald JM, Sadatsafavi M. Economic and health effect of full adherence to controller therapy in adults with uncontrolled asthma: a simulation study. J Allergy Clin Immunol. 2014;134(4):908-15.
7. Medicaid.gov. Eligibility. Accessed October 10, 2021. https://www.medicaid. gov/medicaid/eligibility/index.html

8. Ford ES, Murphy LB, Khavjou O, Giles WH, Holt JB, Croft JB. Total and state-specific medical and absenteeism costs of COPD among adults aged $\geq 18$ years in the United States for 2010 and projections through 2020. Chest. 2015;147(1):31-45. doi: 10.1378/chest.14-0972

9. Pruitt K. Medicaid coverage of guidelines-based asthma care across 50 states, the District of Columbia, and Puerto Rico, 2016-2017. Prev Chronic Dis. 2018;15:E110. doi: $10.5888 /$ pcd15.180116

10. Vogelmeier CF, Criner GJ, Martínez FJ, et al. Global Strategy for the Diagnosis, Management, and Prevention of Chronic Obstructive Lung Disease 2017 Report: GOLD Executive Summary. Arch Bronconeumol. 2017;53(3):128-49. doi: 10.1016/j.arbres.2017.02.001

11. Centers for Medicare \& Medicaid Services. Medicaid Drug Spending Dashboard. Updated December 22, 2020. Accessed October 10, 2021. https://www. cms.gov/Research-Statistics-Data-andSystems/Statistics-Trends-and-Reports/ Information-on-Prescription-Drugs/ Medicaid

12. Singh D, Agusti A, Anzueto A, et al. Global Strategy for the Diagnosis, Management, and Prevention of Chronic Obstructive Lung Disease: the GOLD Science Committee report 2019. Eur Respir J. 2019;53(5):1900164. doi: 10.1183/13993003.00164-2019

13. Horak F, Doberer D, Eber E, et al. Diagnosis and management of asthma statement on the 2015 GINA Guidelines. Wien Klin Wochenschr. 2016;128(15-16): 541-54. doi: 10.1007/s00508-016-1019-4
14. Sanders GD, Neumann PJ, Basu A, et al. Recommendations for conduct, methodological practices, and reporting of cost-effectiveness analyses: Second Panel on Cost-Effectiveness in Health and Medicine. JAMA. 2016;316(10):1093-103. doi: 10.1001/jama.2016.12195

15. Python Software Foundation. The Python Language Reference - Python 3.6.15 documentation. Accessed October 31, 2021. https://docs.python.org/3.6/ reference/

16. Aalbers R, Vogelmeier C, Kuna P. Achieving asthma control with ICS/ LABA: a review of strategies for asthma management and prevention. Respir Med. 2016;111:1-7. doi:10.1016/j.rmed.2015.11.002

17. Miravitlles M, Andreu I, Romero Y, Sitjar S, Altés A, Anton E. Difficulties in differential diagnosis of COPD and asthma in primary care. Br J Gen Pract. 2012;62(595):e68-e75. doi:10.3399/ bjgp12X625111

18. Liang TZ, Chao JH. Inhaled corticosteroids. In: StatPearls. StatPearls Publishing 2021. Accessed July 26, 2021. http://www. ncbi.nlm.nih.gov/books/NBK470556/

19. Feldman WB, Gagne JJ, Kesselheim AS. Trends in Medicare Part D inhaler spending, 2012-2018. Ann Am Thorac Soc. 2021;18(3):548-50. doi:10.1513/ AnnalsATS.202008-1082RL

20. Statista. Medicaid enrollment 19662019. Accessed July 26, 2021. https:// www.statista.com/statistics/245347/ total-medicaid-enrollment-since-1966/

21. Purvis L, Schondelmeyer S. Rx Price Watch Report. Trends in retail prices of brand name prescription drugs widely used by older Americans, 2006 to 2020. AARP Public Policy Institute, June 2021. doi:10.26419/ppi.00143.001 